\title{
TRADUCCIÓN DEL RESUMEN DE LA OCTAVA EDICIÓN DEL SI BROCHURE ${ }^{1}$
}

Carlos Paniagua Vásquez.

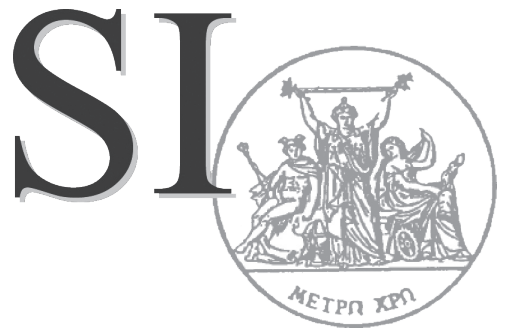

El Sistema Internacional de Unidades, el SI, es el acuerdo internacional, para expresar las medidas en todos los niveles de precisión y en todas las áreas de la ciencia, la tecnología y el quehacer diario

El prototipo internacional del kilogramo, $\mathcal{K}$, único artefacto remanente usado para definir una unidad base del SI.

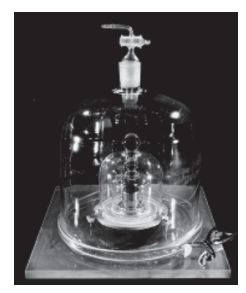

\section{UNIDADES BASE DEL SI}

Existen dos clases de unidades del SI, las unidades base y las unidades derivadas. Las siete unidades base del SI, enumeradas a continuación con sus correspondientes magnitudes base, dan la referencia usada para definir todas las unidades de medida del Sistema Internacional.

\section{LAS SIETE UNIDADES BASE DEL SI}

magnitud

Unidad base, símbolo: definición de la unidad

\section{longitud}

metro, m: el metro es la longitud de la trayectoria recorrida por la luz en el vacío durante un intervalo de tiempo de 1/299 792458 de segundo.

masa

kilogramo, kg: el kilogramo es la unidad de masa; es igual a la masa prototipo internacional del kilogramo.

\section{tiempo}

segundo, s: el segundo es la duración de 9192631770 períodos de la radiación correspondiente a la transición entre los dos niveles hiperfinos del estado fundamental del átomo de cesio 133.

\section{corriente eléctrica}

ampère, A : el ampère es la intensidad de una corriente constante que, mantenida en dos conductores paralelos, rectilíneos, de longitud infinita y de sección circular despreciable y colocados a una distancia de 1 metro el uno del otro en el vacío, producirán entre estos conductores una fuerza igual a $2 \times 10^{-7}$ newton por metro de longitud.

\section{temperatura termodinámica}

kelvin, K: el kelvin, la unidad de temperatura termodinámica, es la fracción 1/273,16 de la temperatura termodinámica del punto triple del agua. 


\section{cantidad de materia}

mole, mol: la mole es la cantidad de materia de un sistema conteniendo tantas entidades elementales como átomos hay en 0,012 kilogramos de carbono 12.

Cuando se emplea la mole, las entidades elementales deben ser especificadas y pueden ser átomos, moléculas, iones, electrones, u otras partículas o grupos específicos de estas partículas.

\section{intensidad luminosa}

candela, cd: la candela es la intensidad luminosa, en una dirección dada, de una fuente que emite radiación monocromática de frecuencia de $540 \times 10^{12}$ hertz y que tiene una intensidad radiante en esa dirección de 1/683 watt por estereorradián.

\section{UNIDADES DERIVADAS}

Las unidades derivadas son definidas como el producto de potencias de la unidades base y son usadas para medir las magnitudes derivadas.

\begin{tabular}{|c|c|}
\hline $\begin{array}{c}\text { Magnitud } \\
\text { derivada, símbolo }\end{array}$ & $\begin{array}{c}\text { Unidad derivada, } \\
\text { símbolo }\end{array}$ \\
\hline área, $A$ & metro cuadrado, $\mathrm{m}^{2}$ \\
\hline volumen, $V$ & metro cúbico, $\mathrm{m}^{3}$ \\
\hline velocidad, $v$ & metro por segundo, $\mathrm{m} / \mathrm{s}$ \\
\hline aceleración, $a$ & metro por segundo cuadrado, $\mathrm{m} / \mathrm{s}^{2}$ \\
\hline densidad de masa, $\rho$ & kilogramo por metro cúbico, $\mathrm{kg} / \mathrm{m}^{3}$ \\
\hline densidad de corriente, $j$ & ampère por metro cuadrado, $\mathrm{A} / \mathrm{m}^{2}$ \\
\hline $\begin{array}{l}\text { intensidad del campo } \\
\text { magnético, } H\end{array}$ & ampère por metro, $\mathrm{A} / \mathrm{m}$ \\
\hline concentración, $c$ & mole por metro cúbico, $\mathrm{mol} / \mathrm{m}^{3}$ \\
\hline luminancia, $L_{v}$ & candela por metro cuadrado, $\mathrm{cd} / \mathrm{m}^{2}$ \\
\hline dice de refracción, & el número) \\
\hline
\end{tabular}

Magnitudes adimensionales, llamadas también magnitudes de dimensión uno, son definidas como la razón de dos magnitudes de la misma clase (por ejemplo, el índice de refracción, en la tabla a la izquierda, es la razón de dos velocidades). Así la unidad de una magnitud adimensional es la razón de dos unidades SI idénticas, igual a uno. Al expresar los valores de las magnitudes adimensionales la unidad uno, 1 , se omite.

Algunas unidades derivadas tienen un nombre especial, para compactar la expresión de combinaciones de unidades base que son usadas frecuentemente. Existen 22 nombres aprobados para unidades derivadas para usar en el SI.

Aunque el hertz y el becquerel son ambos iguales al recíproco del segundo, el hertz se usa únicamente para fenómenos cíclicos y el bequerel para procesos estocásticos de decaimiento radioactivo.

La unidad de la temperatura Celsius es el grado Celsius, ${ }^{\circ} \mathrm{C}$, que es de la misma magnitud que el kelvin, K. La magnitud temperatura Celsius, $t$, está relacionada con la temperatura termodinámica, $T$, por la ecuación:

$t /{ }^{\circ} \mathrm{C}=T / \mathrm{K}-273,15$.

\section{MÚLTIPLOS Y SUBMÚLTIPLOS DECIMALES DE LAS UNIDADES DEL SI}

Un conjunto de prefijos de múltiplos y submúltiplos se han adoptado para usar con las unidades SI. Pueden ser usados con cualquier unidad base y con cualesquiera de la unidades derivadas con nombres especiales.

Cuando se usan prefijos, el nombre del prefijo y el de la unidad se combinan para formar una sola palabra, similarmente el símbolo del prefijo y de la unidad son escritos sin espacio para formar un solo símbolo, que puede ser elevado a cualquier potencia. 
Prefijos del SI

$\begin{array}{cccccc}\text { Factor } & \text { Nombre } & \text { Símbolo } & \text { Factor } & \text { Nombre } & \text { Símbolo } \\ 10^{1} & \text { deca } & \text { da } & 10^{-1} & \text { deci } & \mathrm{d} \\ 10^{2} & \text { hecto } & \mathrm{h} & 10^{-2} & \text { centi } & \mathrm{c} \\ 10^{3} & \text { kilo } & \mathrm{k} & 10^{-3} & \text { mili } & \mathrm{m} \\ 10^{6} & \text { mega } & \mathrm{M} & 10^{-6} & \text { micro } & \mu \\ 10^{9} & \text { giga } & \mathrm{G} & 10^{-9} & \text { nano } & \mathrm{n} \\ 10^{12} & \text { tera } & \mathrm{T} & 10^{-12} & \text { pico } & \mathrm{p} \\ 10^{15} & \text { peta } & \mathrm{P} & 10^{-15} & \text { femto } & \mathrm{f} \\ 10^{18} & \text { exa } & \mathrm{E} & 10^{-18} & \text { atto } & \mathrm{a} \\ 10^{21} & \text { zetta } & \mathrm{Z} & 10^{-21} & \text { zepto } & \mathrm{z} \\ 10^{24} & \text { yotta } & \mathrm{Y} & 10^{-24} & \text { yocto } & \mathrm{y}\end{array}$

Magnitud derivada
ángulo plano
ángulo sólido
frecuencia
fuerza
presión, tensión
energía, trabajo cantidad de
calor
potencia, flujo radiante
carga eléctrica
diferencia de potencial
eléctrico
capacitancia
resistencia eléctrica
conductancia eléctrica
flujo magnético
densidad de flujo magnético
inductancia
temperatura Celsius
flujo luminoso
actividanancia
dosis absorbida equivalente
adítica

Unidades derivadas con nombres especiales del SI

\begin{tabular}{|c|c|c|}
\hline $\begin{array}{c}\text { Nombre de la unidad } \\
\text { derivada }\end{array}$ & $\begin{array}{c}\text { Símbolo de la } \\
\text { unidad }\end{array}$ & Expresión en unidades bas \\
\hline radián & $\mathrm{rad}$ & $\mathrm{m} / \mathrm{m}=1$ \\
\hline estereoradián & $\mathrm{sr}$ & $\mathrm{m}^{2} / \mathrm{m}^{2}=1$ \\
\hline hertz & $\mathrm{Hz}$ & $\mathrm{s}^{-1}$ \\
\hline newton & $\mathrm{N}$ & $\mathrm{m} \cdot \mathrm{kg} \cdot \mathrm{s}^{-2}$ \\
\hline pascal & $\mathrm{Pa}$ & $\mathrm{N} / \mathrm{m}^{2}=\mathrm{m}^{-1} \cdot \mathrm{kg} \cdot \mathrm{s}^{-2}$ \\
\hline joule & $\mathrm{J}$ & $\mathrm{N} \cdot \mathrm{m}=\mathrm{m}^{2} \cdot \mathrm{kg} \cdot \mathrm{s}^{-2}$ \\
\hline watt & W & $\mathrm{J} / \mathrm{s}=\mathrm{m}^{2} \cdot \mathrm{kg} \cdot \mathrm{s}^{-3}$ \\
\hline coulomb & $\mathrm{C}$ & $\mathrm{s} \cdot \mathrm{A}$ \\
\hline volt & $\mathrm{V}$ & $\mathrm{W} / \mathrm{A}=\mathrm{m}^{2} \cdot \mathrm{kg} \cdot \mathrm{s}^{-3} \cdot \mathrm{A}^{-1}$ \\
\hline farad & $\mathrm{F}$ & $\mathrm{C} / \mathrm{V}=\mathrm{m}^{-2} \cdot \mathrm{kg}^{-1} \cdot \mathrm{s}^{4} \cdot \mathrm{A}^{2}$ \\
\hline ohm & $\Omega$ & $\mathrm{V} / \mathrm{A}=\mathrm{m}^{2} \cdot \mathrm{kg} \cdot \mathrm{s}^{-3} \cdot \mathrm{A}^{-2}$ \\
\hline siemens & $\mathrm{S}$ & $\mathrm{A} / \mathrm{V}=\mathrm{m}^{-2} \cdot \mathrm{kg}^{-1} \cdot \mathrm{s}^{3} \cdot \mathrm{A}^{2}$ \\
\hline weber & $\mathrm{Wb}$ & $\mathrm{V} \cdot \mathrm{s}=\mathrm{m}^{2} \cdot \mathrm{kg} \cdot \mathrm{s}^{-2} \cdot \mathrm{A}^{-1}$ \\
\hline tesla & $\mathrm{T}$ & $\mathrm{Wb} / \mathrm{m}^{2}=\mathrm{kg} \cdot \mathrm{s}^{-2} \cdot \mathrm{A}^{-1}$ \\
\hline henry & $\mathrm{H}$ & $\mathrm{Wb} / \mathrm{A}=\mathrm{m}^{2} \cdot \mathrm{kg} \cdot \mathrm{s}^{-2} \cdot \mathrm{A}^{-2}$ \\
\hline grado Celsius & ${ }^{\circ} \mathrm{C}$ & $\mathrm{K}$ \\
\hline lumen & $\operatorname{lm}$ & $\mathrm{cd} \cdot \mathrm{sr}=\mathrm{cd}$ \\
\hline $\operatorname{lux}$ & $1 \mathrm{x}$ & $\mathrm{lm} / \mathrm{m}^{2}=\mathrm{m}^{-2} \cdot \mathrm{cd}$ \\
\hline becquerel & $\mathrm{Bq}$ & $\mathrm{s}^{-1}$ \\
\hline gray & Gy & $\mathrm{J} / \mathrm{kg}=\mathrm{m}^{2} \cdot \mathrm{s}^{-2}$ \\
\hline sievert & Sv & $\mathrm{J} / \mathrm{kg}=\mathrm{m}^{2} \cdot \mathrm{s}^{-2}$ \\
\hline katal & kat & $\mathrm{s}^{-1} \cdot \mathrm{mol}$ \\
\hline
\end{tabular}


Por ejemplo, debemos escribir kilómetro, km; microvolt, $\mu \mathrm{V} ; 50 \mathrm{~V} / \mathrm{cm}=50 \mathrm{~V}\left(10^{-2} \mathrm{~m}^{-1}=\right.$ $5000 \mathrm{~V} / \mathrm{m}$.

El kilogramo, kg, es una excepción por razones históricas, porque es una unidad base cuyo nombre incluye el prefijo kilo.

Los múltiplos y submúltiplos del kilogramo se escriben combinando los prefijos con el gramo: así escribimos miligramo, $\mathrm{mg}$, no microkilogramo, $\mu \mathrm{kg}$.

\section{UNIDADES FUERA DEL SI}

El SI es el único sistema de unidades que es reconocido universalmente, lo que facilita el establecer un diálogo a nivel mundial. Sin embargo, por razones históricas algunas unidades que no son del SI todavía son usadas ampliamente para llenar las necesidades de grupos con intereses especiales, o porque no existe una alternativa SI conveniente. Siempre existirá la prerrogativa del científico para usar las unidades que mejor se ajusten a sus propósitos. Sin embargo, cuando se usan unidades fuera del SI, el factor de conversión a las unidades SI debe citarse (con algunas excepciones de unidades fuera del SI muy familiares).

Algunas de las más importantes y familiares unidades fuera del SI son el minuto, símbolo min, la hora, símbolo h, y el día, símbolo d, como unidades de tiempo.

\section{USO DEL SI PARA EXPRESAR VALORES DE MAGNITUDES}

El valor de una magnitud se escribe como el producto de un número por una unidad. Debe dejarse siempre un espacio entre el número y la unidad. El valor numérico depende de la unidad que se escoja, así el mismo valor de una magnitud puede tener diferentes valores numéricos cuando se expresa en unidades diferentes. Por ejemplo, el valor de la velocidad de una bicicleta puede ser $v=5,0 \mathrm{~m} / \mathrm{s}=18 \mathrm{~km} / \mathrm{h}$.

Símbolos de magnitudes se escriben con tipo itálica (inclinado). Pueden usarse letras mayúscula o minúsculas.

Símbolo de las unidades se escriben en tipo romano (recto) independientemente del tipo de letra que se esté usando en el texto. Son entidades matemáticas y no abreviaturas; nunca van seguidas de punto (excepto si es al final de una oración). Se escriben con minúscula, excepto que la primera letra es mayúscula cuando el nombre de la unidad proviene de un individuo (por ejemplo, ampère, A; kelvin, K; hertz, Hz; coulomb, C; pero metro, m; segundo s).

El uso de la forma correcta de los símbolos de las unidades es obligatorio.

Para cada magnitud existe solo una unidad SI. Sin embargo, la misma unidad SI puede expresar el valor de diferentes magnitudes. Por ejemplo el $\mathrm{J} / \mathrm{K}$ es la unidad SI para la capacidad calórica y para la entropía. Por la tanto, es importante no usar solamente la unidad para especificar la magnitud.

El marcador decimal puede ser tanto la coma (,) como el punto (.) dependiendo del lenguaje del texto circundante y la legislación del país.

Cuando un número tiene muchos dígitos, se establece que deben separarse en grupos de tres a partir del marcador decimal (a la derecha y a la izquierda) para facilitar la lectura, usando un espacio, no deben usarse ni la coma ni el punto.

Como los símbolos de las unidades son entidades matemáticas, deben ser tratados por la reglas ordinarias del álgebra. Por ejemplo la ecuación $T=293 \mathrm{~K}$ puede ser escrita $T / \mathrm{K}=293$. Este procedimiento es descrito como cálculo de magnitudes o álgebra de magnitudes.

Es a menudo conveniente usar la razón de una magnitud sobre su unidad en el encabezado de las columnas de una tabla, y para identificar los ejes de un gráfico, así los valores de la tabla o las divisiones de los ejes del gráfico son simplemente números. 
NOTA

1. Este texto es un resumen de la octava edición del SI Brochure, preparado por el Comité Consultatif des Unités (CCU) del Comité International des Poids et Mesures (CIPM), y es publicado por el BIPM.

Traducido del original en inglés publicado en marzo del 2006, por el Lic. Carlos Paniagua Vásquez del Laboratorio de Metrología, Normalización y Calidad (LABCAL), del Instituto de Investigaciones en Ingeniería, Universidad de Costa Rica.
Para mayor información visite el sitio Web del Bureau International des Poids et Mesures, BIPM, en www.bipm.org

\section{SOBRE EL AUTOR}

\section{Carlos Paniagua Vásquez}

Licenciado en Química

Investigador Invitado del Instituto de Investigaciones en Ingeniería, Universidad de Costa Rica

Teléfono: (506) 2202-6159

Facsímil: (506) 2253-4617

Correo electrónico: carlosbpaniagua@gmail.com 
